Oral presentation

Open Access

\title{
Abstract withdrawn
}

from 2005 International Meeting of The Institute of Human Virology Baltimore, USA, 29 August - 2 September 2005

Published: 8 December 2005

Retrovirology 2005, 2(SuppI I):SI46 doi: 10. I 186/I742-4690-2-SI-SI46 